phylogeny to detect HIV clusters has highlighted acute infection in some studies (especially among MSM), but such work has been primarily retrospective; real-time use of viral phylogeny for HIV prevention has not yet been implemented. Ongoing community based "test and treat" trials in Africa may help determine the importance of acute infection in the centre of the pandemic. The outstanding question for public policy is what degree of emphasis to place on detection and treatment of acute and early HIV, a question that can only be addressed with further empirical results and cost/benefit analysis.

\section{PL05.2 WHY STI-ASSOCIATED GENITAL TRACT INFLAMMATION STILL MATTERS IN HIV TRANSMISSION}

Jo-Ann Passmore. Institute of Infectious Disease and Molecular Medicine (IDM), University of Cape Town, Centre for the AIDS Programme of Research in South Africa (CAPRISA), National Health Laboratory Service, South Africa

\subsection{6/sextrans-2015-052270.14}

Women in Africa, especially young women, have very high HIV incidence rates that cannot be fully explained by behavioural risks. In the setting of syndromic management for sexually transmitted infections (STIs) and bacterial vaginosis (BV), the influence of these, particularly asymptomatic infections, on $\mathrm{CD}^{+}{ }^{+} \mathrm{T}$ cell activation and inflammation in the genital tracts of adolescents from South Africa urgently needs to be addressed. The influence of genital inflammation on HIV acquisition in this group will be discussed. Our study found that HIV seroconversion was associated with raised genital inflammatory cytokines (including chemokines MIP-1 $\alpha$, MIP-1 $\beta$ and IP-10). The risk of HIV acquisition was significantly higher in women with evidence of genital inflammation, defined by at least 5 of 9 inflammatory cytokines being raised [OR 3.2; 95\% confidence interval 1.37.9]. Genital cytokine concentrations were persistently raised (for about one year before infection), with no readily identifiable cause despite extensive investigation of several potential factors, including STIs and systemic cytokines. Adolescents (median 18 years) had significantly higher frequencies of activated $\mathrm{CD}^{+}{ }^{+} \mathrm{T}$ cells $\left(\mathrm{CD} 38^{+}, \mathrm{HLADR}^{+}, \mathrm{CD}_{3} 8^{+} \mathrm{HLADR}^{+}\right)$from cervical cytobrushes than adults, although CCR5 expression was higher in adults. STIs and BV prevalence was very high in certain areas of South Africa, with 71\% of adolescents having $>1$ STI and/or BV, and $42 \%$ being C. trachomatis positive. Adolescents with an STI had higher frequencies of activated and proliferating T-cells compared to those with no STI/BV. Higher cervical T-cell activation marker expression was directly associated with increased genital cytokine profiles. Our data suggests that elevated genital concentrations of HIV target cell-recruiting chemokines and a genital inflammatory profile contributes to the high risk of HIV acquisition in these African women. In adolescents, heightened levels of genital immune activation and inflammation, partly due to the presence of asymptomatic STIs/BV, could increase their risk for HIV infection.

\section{PL05.3 HIV AND SYPHILIS: A SYNDEMIC WITH NO END IN SIGHT}

Khalil Ghanem. Associate Professor of Medicine, Johns Hopkins University School of Medicine, Baltimore, USA

10.1136/sextrans-2015-052270.15
Syphilis rates are increasing in many parts of the world. HIVinfected persons, particularly men who have sex with men (MSM), have been affected disproportionately. In a recent study, $15 \%$ of patients attending New York City STD clinics who were diagnosed with syphilis subsequently became HIV-infected. Several questions about the optimal management of syphilis in HIVinfected persons remain unanswered and continue to elicit controversy, yet none of these are responsible for this persistent syndemic. There are, however, many questions whose answers may critically impact the control of these infections: What is the role of novel point-of-care syphilis tests? Is there a role for syphilis pre-exposure prophylaxis and male circumcision? How will PrEP impact the rates of syphilis? Seventy years following the introduction of a cure, and over 30 years after the discovery of HIV, syphilis continues to present formidable challenges to public health.

\section{Plenary Session PL06}

\section{Wednesday 16 September 2015}

\section{$3.45 \mathrm{pm}-5.15 \mathrm{pm}$}

\section{PL06.1 LEGAL AND HUMAN RIGHTS DIMENSIONS OF HIV AND STI: HISTORICAL BACKGROUND AND POLICY IMPLICATIONS}

Carlos Cáceres. Professor of Public Health and Director, Center for Interdisciplinary Studies in Sexuality, AIDS and Society, Universidad Peruana Cayetano Heredia, Lima, Peru

\subsection{6/sextrans-2015-052270.16}

The degree of regulation of sexuality has been very diverse through times and cultures. Christianity regulated it heavily, and many of its views became part of the European legal tradition. The last thirty years have seen considerable changes in Western culture, including the emergence of social movements around gender equity, sexual diversity, and HIV. In connection with the struggles of these movements as well as other social and political changes, there has been considerable progress in the situation of women and people with non-heterosexual identities, although in this case there has been a greater range of changes, including some on the negative side, such as criminalization. Finally, the global HIV movement made significant changes in the relationship between scientists, doctors, community and regulatory agencies, and led a crusade to expand access to treatment and prevention.

Because HIV is primarily sexually transmitted, and more prevalent in socially excluded groups in countries without generalized epidemics, AIDS was doubly stigmatized: as a deadly and mysterious disease, and as an indicator of social exclusion. Predictably, HIV stigma led to discrimination in access to services. In most of the world, the promotion of condom use did reduce, but never eliminated HIV transmission. Such finding brought a focus both to cultural differences and to structural vulnerability. HIV is clearly more frequent among socially excluded people, who need public interventions to reduce such vulnerability. In its history of more than three decades, HIV has elicited a variety of responses in legislative and regulatory frameworks, both positive and negative, some of which will be discussed.

The history of the response to STIs, with emphasis on the modern HIV epidemic shows the growing relationship between public health, public policy and legislation, and the potential 